Musées, Patrimoine et Culture scientifiques et techniques

$108 \mid 2006$

novembre-décembre 2006

\title{
Les systèmes de projection des planétariums : de l'optomécanique au numérique
}

\author{
Lionel Ruiz et Agnès Acker
}

\section{OpenEdition \\ Journals}

Édition électronique

URL : http://journals.openedition.org/ocim/1316

DOI : $10.4000 /$ ocim. 1316

ISSN : 2108-646X

Éditeur

OCIM

Édition imprimée

Date de publication : 25 novembre 2006

ISSN : 0994-1908

Référence électronique

Lionel Ruiz et Agnès Acker, « Les systèmes de projection des planétariums : de l'optomécanique au numérique », La Lettre de l'OCIM [En ligne], 108 | 2006, mis en ligne le 12 mars 2014, consulté le 23 avril 2019. URL : http://journals.openedition.org/ocim/1316 ; DOI : 10.4000/ocim.1316 


\section{Les systèmes de projection des planétariums : de l'optomécanique au numérique}

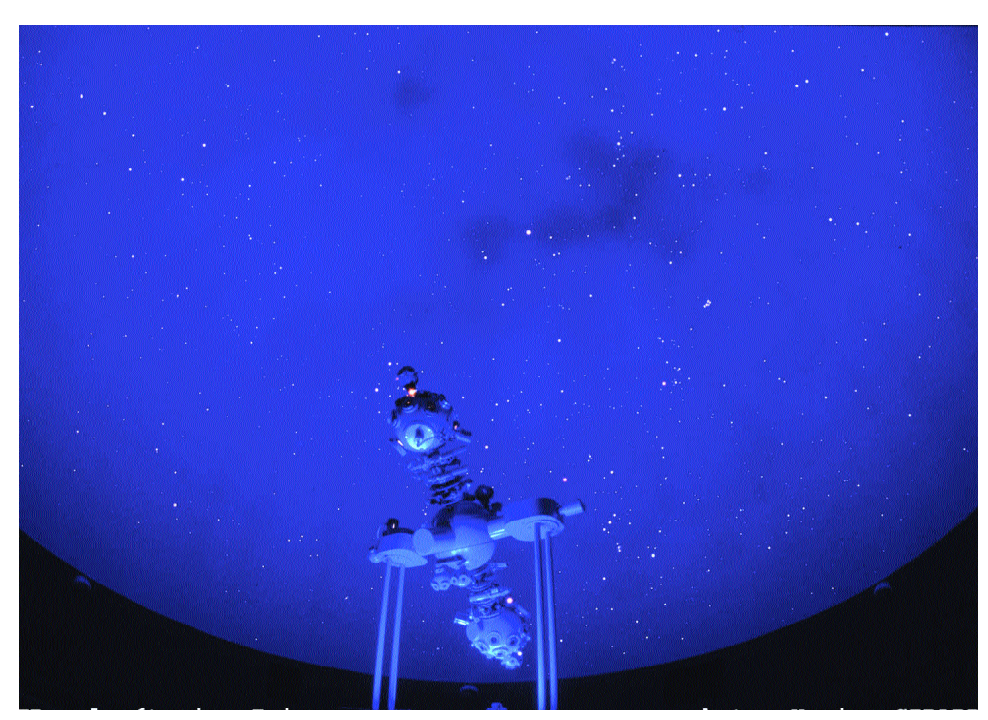

Le planétarium optomécanique de Ludiver : le projecteur Zeiss ZKP3 sous une voûte de 10 mètres à Ludiver à La Hague. Comme avec tous les projecteurs Zeiss, la qualité de projection des étoiles fascine autant que la machine qui les produit. Souvent après les séances, une fois les lumières rallumées, les spectateurs admirent pendant de longues minutes cette belle mécanique. (๑) Xavier Girard

*Lionel Ruiz est médiateur scientifique à l'association Andromède Observatoire de Marseille 2 place Le Verrier 13248 Marseille cedex 04 téléphone + 33495044118 lionel.ruiz@netcourrier.com

\section{Lionel Ruiz et Agnès Acker *}

Après avoir exposé les différents systèmes de projection aujourd'hui en usage dans les planétariums, les auteurs s'intéressent plus particulièrement au développement récent du système numérique, analysant les avantages et les inconvénients de cette technologie dont l'utilisation est de plus en plus répandue.

Le premier système de projection du ciel étoilé fut introduit en 1915 par Oskar von Miller et Walter Bauersfeld, créateur de la firme Zeiss. Le premier spectacle présenté au public en 1925 provoqua une grande admiration pour le miracle technologique Zeiss, cependant que vers 1950 les petits projecteurs Spitz 512 enrichissaient les salles de classe aux ÉtatsUnis. Puis, à partir de 1965, ce fut l'ère des théâtres multi-images, en harmonie avec la grande époque de la conquête de la Lune, et avec l'intérêt politique pour une communication prestigieuse vers le public. Aux États-Unis, à partir de 1980, les spectacles immersifs avec « allsky » ont peu à peu pris une tournure ludique et " hollywoodienne », avec shows laser et mickey visitant les trous noirs ! En Europe, un rôle éducatif plus austère a prédominé. Le multimedia s'est imposé vers 1990, aboutissant sur toute la planète aux planétariums numériques du $3^{\mathrm{e}}$ millénaire.

\section{Les différents systèmes de projection}

Deux systèmes permettent la projection du ciel étoilé, animé des mouvements diurne, annuel, de précession, et en latitude. Le système optomécanique fut utilisé dès les débuts du planétarium, et équipe encore de nombreux projecteurs. 

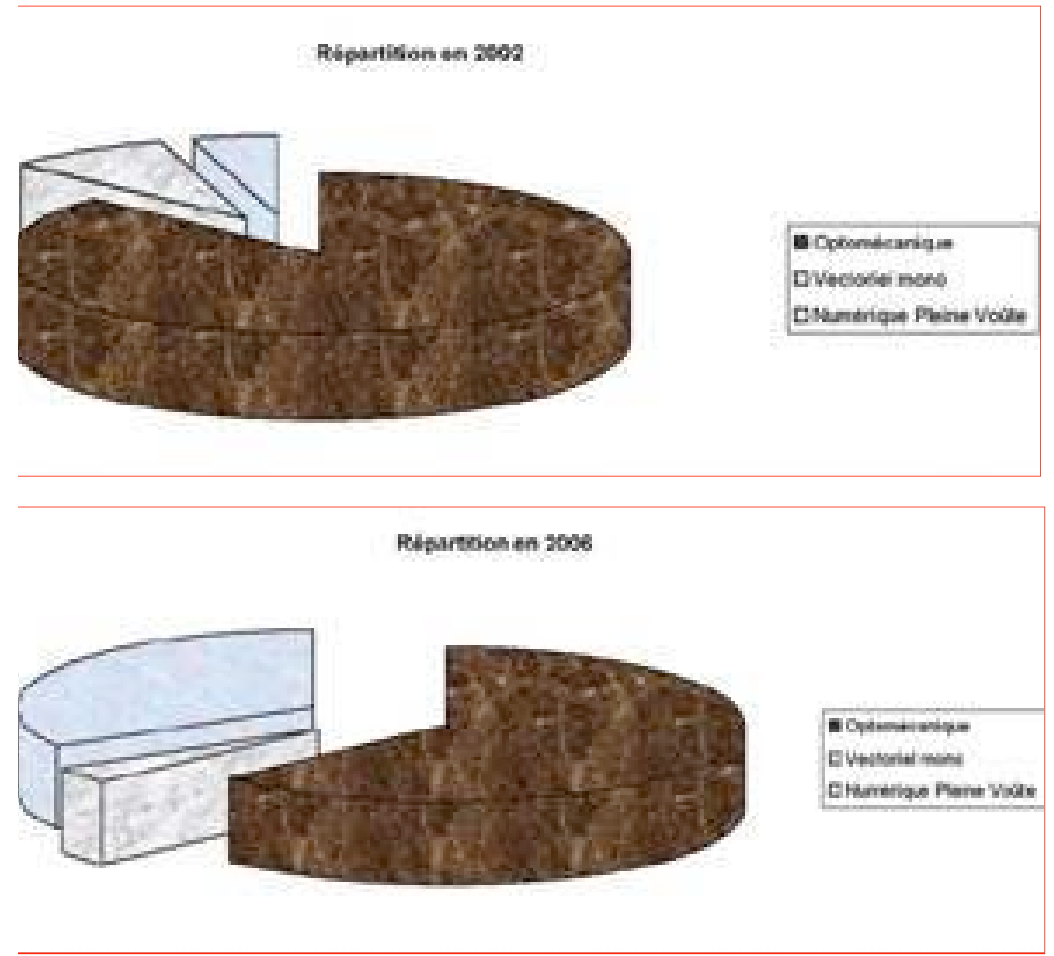

une projection «fenêtrée » sur le dôme, mais imposent l'emploi d'un lecteur de source vidéo : lecteur de DVD, PC, LaserDisc ou CDI (ces deux derniers devenant obsolètes). Les vidéoprojecteurs sont moins bruyants que les projecteurs de diapositives, et leurs séquences défilent sans intervention de l'animateur qui ne fait que les lancer. Mais la vidéo projection a l'inconvénient de projeter des images de faible résolution (1024 x 768 pixels par exemple) dont le fond n'est jamais totalement noir (il faut prévoir des obturateurs mécaniques, masques, filtres).

\section{Les planétariums numériques}

\section{Les projecteurs équipés d'une optique "fisheye»}

Il s'agit d'un projecteur fonctionnant comme un ordinateur dont l'écran serait étendu à la totalité du dôme du planétarium, avec une seule et unique optique, d'où un encombre-

Évolution entre 2002 et 2006 des systèmes de projection (c) Lionel Ruiz

\section{Les systèmes optomécaniques}

Les systèmes optomécaniques sont formés d'une sphère opaque, percée de trous éclairés par une lampe interne, qui peut tourner suivant différents axes pour reproduire les mouvements apparents de la voûte étoilée. On peut y adjoindre des projecteurs annexes pour montrer les planètes, la Lune, l'écliptique, l'équateur, le méridien central, les points cardinaux, les étoiles filantes, les constellations, le cercle de précession, le système solaire héliocentrique...

\section{Allsky : un effet immersif}

Des effets supplémentaires sont obtenus par l'adjonction de projecteurs de diapositives. Une couverture totale de la voûte peut être obtenue par le système de projection pleine voûte (allsky). Six projecteurs de diapositives couvrent chacun $1 / 6^{e}$ de la voûte, pour y reconstituer une image entière couvrant toute la demi sphère : le spectateur se trouve alors plongé dans un paysage cosmique, nébuleuse ou galaxie, ou au pied d'un immense observatoire ou d'une pyramide, ou au cœur d'une forêt. Ces effets sont souvent étonnants et permettent de rythmer un spectacle.

\section{Les projecteurs vidéo}

Généralement au nombre de deux dans le cas d'une salle non orientée, les vidéo projecteurs permettent ment réduit et une vision "englobante ». Mais la projection est déformée en périphérie de projection, et les matrices de projection couleur manquent de résolution - sauf pour les systèmes vectoriels monochromatiques (Digistar I et II de Evans \& Sutherland). De plus, la projection couleur s'avère très limitée pour de grandes salles, allant de $768 \mathrm{x}$ 768 pixels pour le Vdôme de RSA et le Digitarium de Digitalis Education, à 1280 x 1280 pour des systèmes simple tête de Skyskan/Zeiss.

\section{Les systèmes numériques multi vidéoprojecteurs}

Avec un budget plus conséquent, on peut opter pour une couverture de projection à l'aide de plusieurs vidéoprojecteurs. En multipliant les projecteurs, on augmente la résolution en fragmentant la voûte par morceaux avec des optiques adaptées, mais cela engendre des problèmes de jonction à la limite de chacune des zones de recouvrement - ce qui impose de masquer partiellement ces bords de zone de projection (technique du soft-edge). Les grandes salles ont souvent besoin de doubler les vidéoprojecteurs pour avoir une luminosité de couverture suffisante.

\section{Le système laser RVB}

Hormis un coût difficilement compatible avec tous les budgets, cette solution qui en est actuellement au stade du test de la fiabilité dans le temps s'avérera la technologie irremplaçable en termes de finesse, de luminosité, de contraste et de mise en œuvre dans le monde des planétariums de demain. 


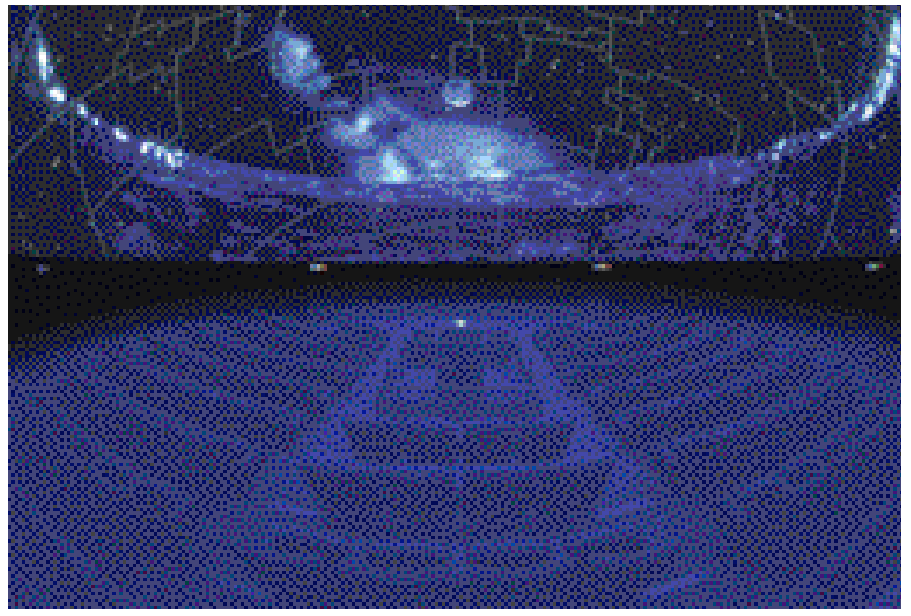

Le Digistar II et les 6 vidéoprojecteurs sous une voûte de 15 mètres au planétarium numérique Galilée à Montpellier : deux images prises successivement au même endroit ont été par la suite juxtaposées : celle des 152 fauteuils de la salle éclairés par les lumières d'ambiance bleues, et l'ensemble de la voûte. Sur la voûte, le Digistar II (au centre de la salle) projette le ciel étoilé et les limites des constellations définies par l'union astronomique internationale, tandis que les 6 vidéoprojecteurs immergent le public au cœur d'un panorama sur Pluton avec juste au dessus de l'horizon, son satellite Charon qui vient de se lever. () Xavier Girard

\section{Avantages et inconvénients du numérique}

\section{Les avantages}

\section{Des effets vidéo sans limites}

Des effets impossibles à produire par les systèmes optomécaniques sont désormais accessibles : mouvements dans le système solaire et la Galaxie, phénomènes de variabilité des étoiles, de contraction des nébuleuses en voie de formation stellaire, d'éjection d'une nébuleuse en fin de vie stellaire... Le numérique permet une dynamique de mouvement et une rapidité de mise en œuvre incomparables.

Toute la zone écran du dôme ou seulement une partie peut être utilisée pour une projection animée. L'intégration dans la voûte est donc parfaite avec une localisation qui peut être étudiée en fonction des explications. L'animation pleine voûte permet ainsi certaines démonstrations dynamiques saisissantes comme la traversée des anneaux de Saturne ou le déplacement à l'intérieur d'une structure tridimensionnelle complexe (comme la Galaxie, les nébuleuses ou les amas stellaires). Cette démarche n'est pas seulement spectaculaire, elle est aussi nécessaire pour les scientifiques eux-mêmes et est enfin disponible pour le grand public.

\section{Une banque d'images bien organisée}

Les sources scientifiques actuelles sont désormais numériques, les transformer en diapositives coûte beaucoup d'énergie. Le simple fait de pouvoir augmenter la banque d'images de façon immédiate permet de coller au plus vite à l'actualité. Avec une banque de données bien organisée, l'animateur peut répondre immédiatement aux questions posées par le public en fin de séance avec des images à l'appui.

\section{Des réglages automatiques et quasi immédiats}

Positionner un projecteur à une date et à une heure précises nécessite un temps mécanique incompressible. Ce n'est pas le cas du numérique qui permet de basculer en une fraction de seconde à la date voulue. Ceci permet de programmer des effets visuels synchronisés sur un temps particulier (temps calé sur un astre par exemple) pour des effets de trace ou de suivi du plus bel effet et dont l'intérêt pédagogique est indéniable.

\section{La pluralité des présentations}

et la variété des sujets

Adapter une salle à un spectacle prend du temps avec un projecteur optomécanique. Avec le numérique, une grande quantité de spectacles peuvent être lancés - y compris pour des domaines autres que l'Astronomie.

\section{Les inconvénients}

\section{Le manque de résolution}

C'est le point majeur que mettent en avant ceux qui sont contre le passage au numérique. Cette critique s'estompera avec le temps - comme ce sera le cas pour les appareils photographiques numériques dont la résolution actuelle est pauvre face à celle des photographies argentiques. Un ensemble de vidéo projecteurs ne sera utile que si le rendu des étoiles permet de reconnaître les constellations, en respectant l'échelle des magnitudes. Ce critère devrait être préalable à toute discussion technique.

Les vidéoprojecteurs n'ont pas un fond noir total mais un fond très légèrement gris quand rien n'est projeté, qui varie selon les types LCD, DLP ou CRT. Le planétarium de Saint-Étienne dispose de vidéo projecteurs de type tri-tubes ; son responsable Cyril Fernandez rapporte qu'ils ont l'avantage d'avoir un noir parfait, mais nécessitent une mise au point pointilleuse qui, à la longue, se perd peu à peu, ce qui, sur des objets aussi ponctuels que des étoiles, devient rapidement très gênant. Pascal Prieur de la Cité de l'Espace à Toulouse reconnaît que le CRT 


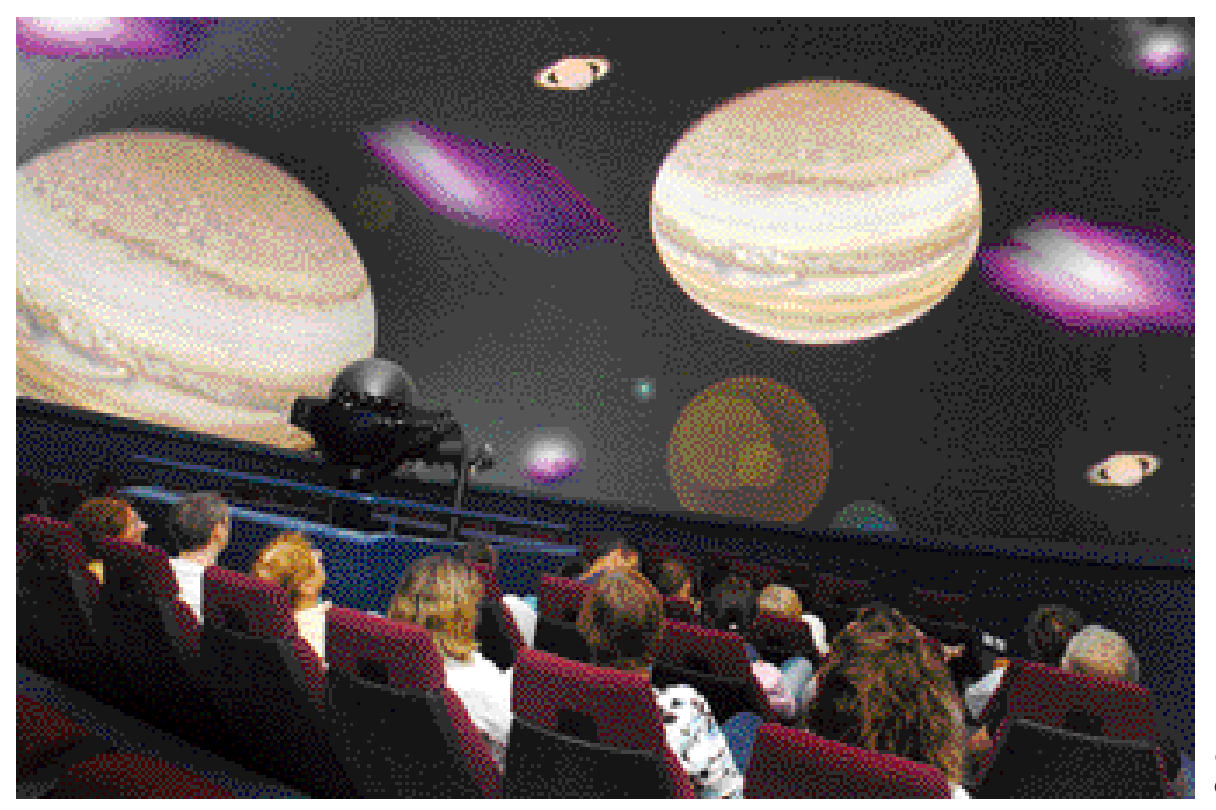

Le planétarium numérique au muséum-jardin des Sciences de Dijon

(c) mairie de Dijon/Ludovic Charron

reste bien le seul à fournir un noir noir, une finesse et une douceur de point (pas de scintillement ni de moirage...), mais il ajoute que le réglage des 12 projecteurs CRT du planétarium de la Cité de l'Espace relève plus de l'exploitation d'une usine à gaz que d'une partie de plaisir.

Il est vrai que du point de vue de la finesse, celle du «vrai » ciel est difficilement reproductible et que même les projecteurs optomécaniques ne sont pas toujours excellents. Pour les projecteurs numériques, jouer sur la luminosité des pixels permet souvent de compenser une résolution déficiente. Mais pour vraiment comparer les performances des 2 types de projecteurs, il faudrait mettre en équation d'une part, la taille de la sphère de projection, la taille des trous, la puissance de la lampe centrale, la dominante de la lampe et d'autre part, la luminosité, le contraste et la résolution.

\section{L'ergonomie manipulatoire}

Appuyer sur dix boutons et manettes n'est pas chose évidente à retrouver dans l'obscurité, mais cliquer sur des centaines d'options et sous-menus à la volée lors d'une séance n'est pas forcément plus aisé. L'ergonomie est primordiale et le logiciel se doit d'être conçu pour un utilisateur qui n'a pas forcément l'étoffe d'un maniaque des boutons, menus déroulants et glissés-déplacés. Surtout que la rapidité d'action est inversement proportionnelle à la précision de pointage. Tous les systèmes ne sont pas à la même enseigne de ce point de vue là.

\section{Les pannes et mises à jour}

Statistiquement, un système optomécanique est plus robuste car de légères pannes peuvent survenir sans provoquer l'annulation des séances. Il nécessite juste des temps de révision et de réparation. Lorsque l'informatique est en panne, plus rien ne marche. D'autre part, les progrès de l'informatique, l'actualité, la meilleure connaissance des modélisations peuvent amener à faire évoluer un système numérique et cela prend du temps. De plus, si un vidéo projecteur est en panne, la maintenance n'est pas instantanée, cela nécessite un bon diagnostic et d'avoir l'élément de remplacement sous la main. On peut donc être contraint d'annuler des séances, car on ne change pas un vidéprojecteur comme un projecteur de diapositives.

\section{Le poids des données}

Pour Lionel Ruiz, un petit planétarium peut s'en sortir aisément au niveau des vidéos car il se contente d'utiliser des résolutions classiques (720 x 576 par exemple). Par contre, lorsqu'il s'agit de travailler avec des séquences pré-calculées en 3072 x 3072 à 30 images par secondes, on atteint vite une capacité excessive à stocker et quasiment aucun fichier vidéo professionnel ne possède cette résolution. Les séquences numériques en 1900 x 1200 sont seulement expérimentales et concernent rarement l'Astronomie. Heureusement, notre œil peut se contenter d'une image de faible résolution dans la mesure où elle n'est pas fixe. D'où la volonté quasi maladive de certains à user de mouvements pour cacher cette faiblesse. À ce sujet, les projecteurs modestes en 768 x 768 sont certes peu convaincants pour afficher des étoiles réalistes, mais en terme de vidéo, le rendu peut être vraiment surprenant. Et gérer un flux de cette résolution est en parfaite adéquation avec les micro-ordinateurs actuels. 


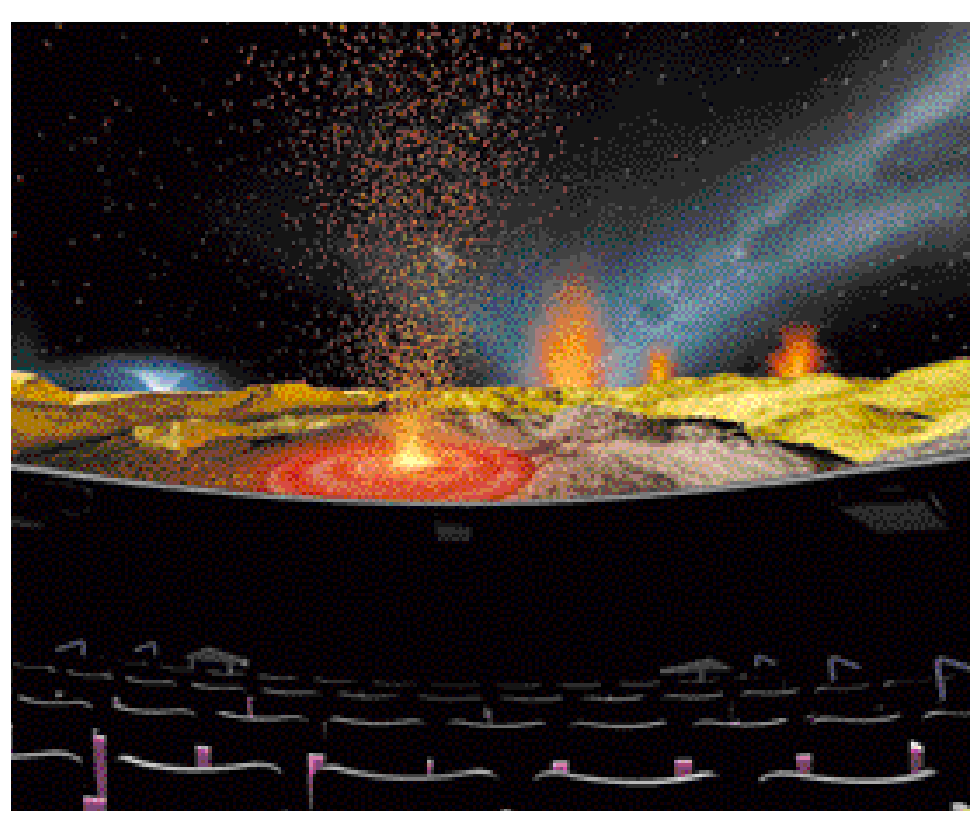

Paysage animé de volcans en video pleine voûte (c) DIGISTAR/Evans \& Sutherland

Pascal Prieur insiste sur le problème posé par l'archivage des données. Une quantité « astronomique » de fichiers est générée par cette nouvelle technologie (45 000 images x 3 étapes de fabrication, soit environ 10 terra octets de calcul pour 30 minutes de spectacle). Si on ne veut pas être vite submergé ou être confronté au dilemme de la perte de données, il faut non seulement anticiper largement l'achat de disques durs, mais aussi prévoir et gérer ce rangement, ou mieux, créer une banque pour échange ou revente de séquences. C'est un sujet qui devrait rapidement intéresser une communauté de planétariums en France ou en Europe.

\section{Le prix du matériel}

C'est peut-être le plus important des inconvénients. Mais les prix évoluent favorablement car le matériel idéal se rapproche de plus en plus du matériel courant commercialisé. Les prix indicatifs (sans coupole) sont d'environ $12000 €$ pour les mobiles optiques, d'environ $15000 €$ pour les mobiles numériques, d'environ 300000 à $800000 €$ pour les systèmes numériques avec DLP ( 5 ou 6 projecteurs) et plus d'un million d'euros avec 6 projecteurs CRT.

\section{Les difficultés de la production}

Programmer l'affichage du ciel, glisser une diapositive dans un carrousel, mettre tel CD dans un lecteur, tout cela prend un temps que l'on peut qualifier d'humain. Pour obtenir un rendu informatique, ce temps peut devenir « astronomique » en termes de calculs. Évidemment, il existe aussi la solution d'acheter des spectacles tout faits, mais leurs prix sont en général eux aussi « astronomiques ».

Pascal Prieur se réjouit de l'apparition (enfin !) d'un vrai format d'échange le «Master Dôme » : format rond (dans un carré), de résolution variable (dont dépend la qualité finale), et du standard au choix 25, 29,97 ou 30 images/seconde. Il est maintenant aisé d'échanger, d'acheter, coproduire des séquences vidéo dôme avec n'importe quel planétarium numérique dans le monde. La conséquence immédiate est la baisse des coûts, ces budgets qui nous soucient tant ! Mais la production d'un film est longue et fastidieuse à réaliser : production d'un modèle $3 \mathrm{D}$, calcul d'une première série d'images, puis d'une deuxième pour arriver au « Master Dôme » et enfin calcul des fichiers vidéo Mpeg HD pour diffusion.

\section{À propos de la production d'un spectacle pleine voûte}

L'équipe du planétarium Galilée de Montpellier constate que, pour chaque nouvelle avancée technologique, se pose le problème de l'intérêt que peut représenter tout nouveau système : vynil/CD, VHS/ Betamax/V2000, analogique/numérique, numérique/argentique, DAT/DCC ... Comment réaliser un spectacle dynamique couvrant la totalité de l'écran hémisphérique, incluant des personnages de manière vivante qui se déplacent d'une partie à l'autre de l'écran ? Un système optomécanique propose certaines solutions : les fondus enchaînés avec des projecteurs de diapositives, l'utilisation d'un projecteur indexable pour donner du mouvement, la projection d'une image vidéo à l'aide d'un ou plusieurs projecteurs sur une portion limitée de l'écran en superposition avec un allsky diapositives. Mais la technique à déployer pour obtenir un résultat probant est très fastidieuse, avec un résultat parfois correct, mais qui a le défaut de se dégrader au fil du temps (problème de différence de teintes entre la vidéo et les diapositives en particulier). Alors que la vidéo pleine voûte permet de créer des séquences spectaculaires, d'y intégrer des personnages en mobilité permanente, d'apporter aisément une dimension dramatique, ou bien d'expliquer un phénomène complexe.

Le planétarium Galilée de Montpellier Agglomération a produit en 2003 ses spectacles en faisant appel à un mélange de deux technologies bien différentes : celle du Digistar 2 qui permet d'avoir une représentation du ciel, et celle du système de vidéo pleine voûte de six vidéoprojecteurs Barco, qui permet de rajouter 


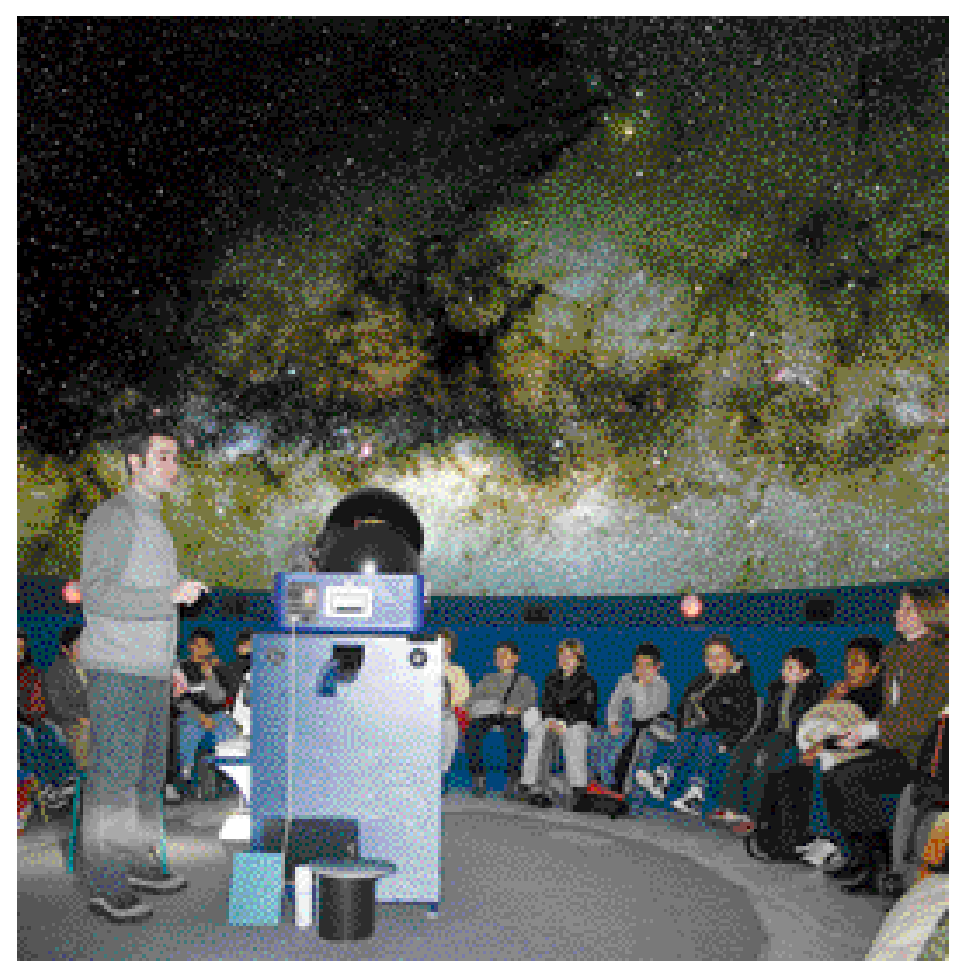

des images pleine voûte, dont de courtes séquences réalisées à l'aide de logiciels 2D et 3D. En 2004 le choix technique et artistique de production de Vacances sur Altaïr a été celui de la seule vidéo pleine voûte, grâce aux avancées technologiques concernant autant les outils de modélisation et de post-production que les temps de calcul ou le stockage (le total des dômes originaux du spectacle approche les 500 Go).

Mais pour un spectacle vidéo pleine voûte, le travail réalisé en amont est beaucoup plus important : en particulier, avant la validation définitive d'un storyboard, il faut par exemple décider du choix du traitement des personnages. Suivant l'option choisie, de la 2D ou de la 3D, la réalisation des décors, des effets spéciaux et du son (bruitage et musique), les temps de fabrication, de calcul et d'archivage vont être appréhendés de manière complètement différente. Et il en découle, quelle que soit l'option choisie, une série de choix techniques et artistiques que l'on se doit d'anticiper du mieux possible, et qui doivent être bien intégrés au planning de production.

En guise de conclusion, Pascal Prieur, responsable audiovisuel de la Cité de l'Espace de Toulouse, reconnaît que tous les planétariums équipés d'un système numérique, petits et grands, vivent la nouvelle aventure numérique de la même façon, avec des avis, des critiques et des impressions relative- ment similaires. À côté des extraordinaires possibilités de présentation et de programmation des spectacles, séduisant les animateurs et le public, tous regrettent de ne pas retrouver la finesse du ciel étoilé qui pour le moment reste l'apanage de l'optique. Ils reconnaissent la difficulté de la manipulation des systèmes numériques, les problèmes récurrents de la maintenance et du stockage de données, et les coûts importants de production ou d'achat, entraînant un budget en forte progression. Ce qui montre l'intérêt croissant d'une politique d'échanges ou/et de production en commun de spectacles - en particulier dans le cadre de l'APLF. Avec le temps, il semble certain que la majeure partie des planétariums «glissera » vers l'image vidéo numérique projetée, grâce à des coûts et des techniques qui vont se démocratiser.

Ce texte a été réalisé avec la contribution des planétariums de Nantes, Toulouse, Montpellier et SaintÉtienne d'après un article paru dans la revue Planétariums 2006. 